\title{
EXISTENCE AND APPLICATIONS OF REMOTE POINTS
}

\author{
BY ERIC K. VAN DOUWEN
}

\author{
Communicated by P. T. Church, May 23, 1977
}

All spaces are completely regular, $X^{*}$ is $\beta X-X$.

A point $p$ of $X^{*}$ will be called a remote point of $X$ if $p \notin \mathrm{Cl}_{\beta X} D$ for every nowhere dense $D \subseteq X$. Fine and Gillman, $[F G]$ showed that $\mathbf{Q}$, the rationals, and $\mathbf{R}$, the reals, have remote points if $C H$ holds; their proof shows that $X$ has remote points if $X$ is separable and not pseudo-compact. We prove the existence of remote points without assuming additional set theoretic axioms, under slightly stronger conditions on $X$.

Recall that a $\pi$-base (or pseudo-base) for a space $X$ is a family $B$ of nonempty open sets such that every nonempty open set of $X$ includes a member of $B$. The $\pi$-weight of a space is the smallest cardinality for a $\pi$-base.

THEOREM A. If $X$ is a nonpseudocompact space with countable $\pi$-weight, then $X$ has $2^{\mathcal{E}}$ remote points.

I originally proved this only for $X=\mathbf{Q}$, improving a technique from [ $\left.\mathbf{v} D_{1}\right]$. I am indebted to Mary Ellen Rudin for showing me how to make my ideas work for $X=\mathbf{R}$. The above theorem is a further improvement.

For applications we need a "pointed" version of extremal disconnectedness.

Definition. If $p \in X$, then $X$ is called extremally disconnected at $p$ if for all disjoint open $U, V \subseteq X, p \notin \bar{U} \cap \bar{V}$.

One can show that $\beta X$ is extremally disconnected at every remote point of $X$. Without much effort one deduces the following theorem. ( $X$ is nowhere locally compact if no point has a compact neighborhood.)

THEOREM B. Let $X$ be a nonpseudocompact space with countable $\pi$ weight.

(a) $\beta X$ is extremally disconnected at some point of $X^{*}$.

(b) If $X$ is nowhere locally compact, $X^{*}$ is extremally disconnected at some point.

Frolík, $[F]$, proved that $X^{*}$ is not homogeneous if $X$ is not pseudocompact. Theorem B can be used to show why $X^{*}$ is not homogeneous, for suitable $X$.

AMS (MOS) subject classifications (1970). Primary 54D35, 54D40; Secondary 54B10, 54G05. 
THEOREM C. Let $X$ be a nowhere locally compact nonpseudocompact separable first countable space. Then $X^{*}$ is not homogeneous because $X^{*}$ is extremally disconnected at some but not at all points.

This applies if e.g. $X=\mathbf{Q}$, or $X=$ \{irrationals $\}$, or $X=$ \{Sorgenfrey line $\}$. As another application we show that certain spaces cannot be factored as a product of spaces without isolated points. The key observation is that $X \times Y$ is not extremally disconnected at any point if $X$ and $Y$ are separable spaces without isolated points.

THEOREM $D . \quad Y$ is not the product of two spaces without isolated points in each of the following two cases:

(a) $Y=\beta X$ for some nonpseudocompact $X$ with countable $\pi$-weight;

(b) $Y=X^{*}$ for some nowhere locally compact nonpseudocompact $X$ with countable $\pi$-weight.

(Actually one does not need the condition on the $\pi$-weight in (a), see [v $\left.D_{2}\right]$, but $I$ do not know if it can be avoided in (b).)

COROLlaRY. $\mathrm{Q}^{*}$ and $\left(\mathrm{Q}^{*}\right)^{\kappa}$ are not homeomorphic, for $\kappa \geqslant 2$.

I do not know if $\left(\mathbf{Q}^{*}\right)^{\mathbf{2}}$ and $\left(\mathbf{Q}^{*}\right)^{\mathbf{3}}$ are nonhomeomorphic.

As yet another application we mention the following curiosities.

EXAMPLE. There is an extremally disconnected space which has a connected compactification.

Indeed, if $X$ is any connected nowhere locally compact separable metrizable space, like $\mathbf{R}^{\omega}$, then the subspace $E$ of all points at which the connected space $\beta X$ is extremally disconnected turns out to be dense in $\beta X$, but then $E$ is extremally disconnected.

For the other application, recall that a space is called $\omega$-bounded if every countable subset has compact closure.

THEOREM E. $\mathbf{R}^{*}$ is the union of three pairwise disjoint dense $\omega$-bounded subspaces.

If one calls a point $p$ of $X^{*}$ a far point of $X$ if $p \notin \mathrm{Cl}_{\beta X} D$ for every closed discrete subset $D$ of $X,\left[\mathrm{v} D_{1}\right]$, then the three subspaces are the remote points of $\mathbf{R}$, the far points of $\mathbf{R}$ which are not remote and the points of $\mathbf{R}^{*}$ which are not even far. Under $\mathrm{CH}$ there is a family of $2^{c}$ such subspaces, $[W]$.

ADDED IN PROOF: If $\kappa$ and $\lambda$ are cardinals with $\kappa>\lambda \geqslant 1$, then $\left(Q^{*}\right)^{\kappa}$ and $\left(Q^{*}\right)^{\lambda}$ are not homeomorphic. The nontrivial proof will appear elsewhere.

\section{REFERENCES}

[vD $]$ E. K. van Douwen, Why certain Čech-Stone remainders are not homogeneous, Colloq. Math. (to appear).

[ $\left.\mathrm{vD}_{2}\right] \longrightarrow$ When $\Pi \beta$ and $\beta \Pi$ are homeomorphic (to appear).

$\left[\mathrm{vD}_{3}\right] \longrightarrow$, Remote points (in preparation).

[F] Z. Frolik, Non-homogenity of $\beta P-P$, Comment. Math. Univ. Carolinae 8 (1967), 705-709. 
[FG] N. J. Fine and L. Gillman, Remote points in $\beta R$, Proc. Amer. Math. Soc. 13 (1962), 29-36. MR 26 \#732.

[W] R. G. Woods, Some $\boldsymbol{K}_{0}$-bounded subsets of Stone-Čech compactifications, Israel J. Math. 9 (1971), 250-256. MR 43 \#3997.

INSTITUTE FOR MEDICINE AND MATHEMATICS, MATHEMATICS BUILDING, OHIO UNIVERSITY, ATHENS, OHIO 45701 